\title{
DIFFERENTIATION OF NEURAL STEM CELLS INTO CELLS OF OLIGODENDROGLIAL LINEAGE
}

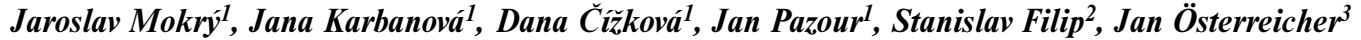 \\ Charles University in Prague, Faculty of Medicine in Hradec Králové and University Hospital Hradec Králové, \\ Czech Republic: Department of Histology and Embryology ${ }^{1}$, Department of Oncology and Radiotherapy ${ }^{2}$ \\ University of Defence, Faculty of Military Medicine in Hradec Králové, Czech Repubic: Department of Radiology ${ }^{3}$
}

\begin{abstract}
Summary: We described three different conditions that induce differentiation of dissociated neural stem cells derived from mouse embryonic CNS. In the first set of experiments, where the cell differentiation was triggered by cell adhesion, removal of growth factors and serum-supplemented medium, only sporadic neuronal and astroglial cells survived longer than two weeks and the latter formed a monolayer. When differentiation was induced in serum-free medium supplemented with retinoic acid, rapid and massive cell death occurred. A prolonged survival was observed in cultivation medium supplemented with serum and growth factors EGF plus FGF-2. One third of the cells did not express cell differentiation markers and were responsible for an increase in cell numbers. The remaining cells differentiated and formed the astrocytic monolayer on which occasional neuronal cells grew. One third of the differentiated phenotypes were represented by cells of oligodendroglial lineage. Differentiation of oligodendroglial cells occurred in a stepwise mechanism because the culture contained all successive developmental stages, including oligodendrocyte progenitors, preoligodendrocytes and immature and mature oligodendrocytes. Maturing oligodendrocytes displayed immunocytochemical and morphological features characteristic of cells that undergo physiological development. The cultivation conditions that supported growth and differentiation of neural stem cells were optimal for in vitro developmental studies and the production of oligodendroglial cells.
\end{abstract}

Key words: Neural stem cells; Mouse; Differentiation; Oligodendroglia; Cell death; In vitro; Phenotypization

\section{Introduction}

Stem cells (SCs) are primitive cells that can self-renew and at the same time generate a differentiated progeny. At early developmental stages, SCs are highly proliferative, giving rise to primordia of developing organs and producing multiple phenotypes that constitute organs and tissues (31). In adulthood, SCs are found in tissues as dormant cells that become activated only when differentiated cells in the tissue are lost and require a replacement. The principal roles SCs play in adult tissues include maintenance of tissue homeostasis and regeneration. Tissue-specific SCs are located in specific microenvironments called "niches" that keep SCs in an undifferentiated state (26). The niche has a complex structure that is constituted by supporting or stromal cells, basal laminae, endothelial cells and components of extracellular matrix. In diseases that reduce the pool of endogenous SCs, the tissue regenerative capacity is diminished or lost, which results in a decrease in its functional capacity. Stem cell therapy represents a novel and promising therapeutic approach to treatment of variety degenerative diseases as multiple sclerosis.
The main requirements for effective cell transplantation involve a source of transplantable cells and proof that the transplanted cells have the capacity to differentiate into cells of a required phenotype. Neural SCs can be obtained from the embryonic or adult CNS. Nowadays, several procedures for the isolation and cultivation of neural SCs have been described. One of the most effective methods is based on exposure of SCs to epidermal growth factor (EGF) and/or fibroblast growth factor-2 (FGF-2) that gives rise to multicellular neurospheres $(24,27-29)$. Each neural SC that is clonogenic can generate a single neurosphere that consists of cells of a clonal origin (25). This clone may comprise more stem cells and progenitor cells which may also have a limited self renewal capacity. As a result, subsequent subcultivation of dissociated neurospheral cells allows to harvest higher amounts of neurospheres. When the dissociated cells are plated at higher cell densities, the resulting neurospheres are heterogeneous and consist of several clones (6). This cultivation procedure is relatively simple and permits a rapid expansion of primitive multipotent neural cells.

The differentiation ability of neurospheral cells can be examined after mitogenic growth factors are withdrawn and 
cells are allowed to adhere to cultivation dishes or after intracerebral transplantation. Under these conditions, the primitive neurospheral cells reveal their multipotency and differentiate into distinct CNS cells. The terminal phenotypes generated by neural SCs in vitro include neuronal, astroglial and oligodendroglial cells (12, 24, 27-29). The production of ependymocytes requires modified conditions (17). Special pre-treatment can be used selectively to suppress or support the generation of desired phenotypes but such approaches are usually more expensive and time consuming.

In this study, we compared three different differentiation protocols and found that the presence of growth factors EGF and FGF-2 prevented cell death, increased cell numbers and supported the survival of undifferentiated cells representing approximately one third of the cells in the assay. Nevertheless most cells underwent spontaneous cell differentiation that favoured cells of astroglial and oligodendroglial lineages. Cultures contained successive stages of oligodendroglial development that expressed characteristic antigenic profiles and displayed morphology indicative of oligodendroglial cells. The mature oligodendrocytes showed all features of physiological differentiation.

\section{Materials and Methods}

\section{Isolation and cultivation of neural stem cells}

The mice used for the experiment were housed in groups of two in a temperature- and humidity-controlled colony room that was maintained on a 12-hour light/dark cycle. Food and water were available ad libitum throughout the experiment. The experiments performed in this study were approved by the Ethical Committee supervising procedures on experimental animals at Charles University, Faculty of Medicine in Hradec Králové, Czech Republic.

Neural SCs were isolated from fetuses of timed pregnant B6,129S-Gt(ROSA)26Sor mice. Forebrains of E15 mouse fetuses were dissected free of meninges and mechanically dissociated by trituration through a fire polished pipette. Single cell suspension of neural progenitors from the fetal brain was plated separately into the culture flasks with untreated surfaces $\left(40,000 / \mathrm{cm}^{2}\right)$. The cultivation medium consisted of Dulbecco's Modified Eagle's Medium/Ham's F12 (1:1; Sigma, Prague, Czech Republic), B27 Supplement without retinyl acetate ( $2 \%$, Gibco, Paisley, UK), $2 \mathrm{mM}$ L-Glutamine (Sigma), $100 \mathrm{U} / \mathrm{ml}$ penicillin and $100 \mu \mathrm{g} / \mathrm{ml}$ streptomycin (Gibco), $10 \mathrm{ng} / \mathrm{ml}$ human recombinant basic fibroblast growth factor (bFGF, PeproTech, London, UK) and $20 \mathrm{ng} / \mathrm{ml}$ mouse recombinant epidermal growth factor (EGF, PeproTech). Neural SCs grew in culture as freefloating neurospheres that were subcultivated by dissociation into single cell suspension two times per week, alternately mechanically or using Trypsin/EDTA (Gibco).

\section{Cell differentiation}

Neural SCs were allowed to differentiate as single cell suspension in I) growth factor free-medium supplemented with fetal calf serum, II) medium composed of Neurobasal medium (Gibco) supplemented with B27 additives, $2 \mathrm{mM}$ L-Glutamine and $1 \mu \mathrm{M}$ all-trans retinoic acid used to enhance differentiation or III) a medium that consisted of full medium with growth factors, used for the generation of neurospheres (described above) supplemented with $10 \%$ fetal calf serum. Cells were allowed to adhere on polyornithine /fibronectin-coated coverslips.

\section{Cytological examination}

Cells in cultures were examined under the phase contrast with the inverted microscope Nikon Eclipse TE300. An Olympus BX50 fluorescent microscope equipped with a DP-70 digital camera was used for examining the coverslips with cells. For immunofluorescence staining, coverglasses with cell monolayer were washed in phosphate buffer containing $0.5 \%$ Triton X-100 (Sigma) treated with $5 \%$ normal goat serum or donkey serum and then incubated overnight with a primary antibody. Mouse monoclonal antibodies were utilized for the detection of glial fibrillary acidic protein (GFAP; GA-5, 1: 400; Sigma), Pan-NF (DA2, FNP7, RmdO20.11, prediluted, Zymed Laboratories, Invitrogen Corp., Paisley, UK), $\beta$-III tubulin (TU-20, 1:20, Exbio, Prague, Czech Republic) and O4 (81, 1:100, Chemicon). Rabbit polyclonal antibodies were used to identify GFAP (1:500, Dako, Glostrup, Denmark) and NG2 (1:200, Chemicon). Following washing, coverslips with cells were incubated with species-specific secondary antibodies for $45 \mathrm{~min}$ conjugated with Cy3, Cy2 (Jackson Immunoresearch; West Grove, PA, USA) or Alexa 488 (Molecular Probes). Slides were mounted in polyvinylalcohol/glycerol with 1,4-diazobicyclo-[2.2.2]-octane (DABCO). To avoid false positivity, parallel coverslips with cells were processed according to the same protocol but primary antibodies were omitted.

\section{Results}

Neurospheral dissociated cells were allowed to adhere to a surface covered with polyornithine/fibronectin and cultured in a medium supplemented with FCS not containing growth factors. Shortly after plating, cells adhered and most cells underwent cell death. The cells that survived were not able to increase their number by proliferation. In the following days, the surviving cells became flattened and tended to form a discontinuous monolayer (Fig. 1A). In gaps left between the flattened astrocytes, multiprocess cells were found. However, these cells died within 14 days and disappeared from the culture. The only cells that survived in the medium grew on surfaces of astrocytic monolayer. Immunocytochemical detection identified only sporadic $\beta$-III tubulin ${ }^{+} / \mathrm{NF}^{+}$neuronal and $\mathrm{O}^{+}$oligodendroglial cells; we identified less than 5 immunoreactive cells per coverslip. The remaining flattened cells that grew in a monolayer were strongly immunoreactive for GFAP. However, these flattened cells did not dispatch cytoplasmic processes. 
When dissociated neurospheral cells were seeded on polyornithine/fibronectin-coated coverslips and grown in serum-free neurobasal medium not containing growth factors but supplemented with retinoic acid, the cells adhered, started to differentiate and astroglial cells formed a complex network. The cells that were observable in gaps between astrocytes expressed first NG2 and in a few days $\mathrm{O} 4$. However, these cells rapidly died and only debris of dead cells was attached to the underlying astrocytic monolayer (Fig. 1B).

The cultivation medium that was permissive for the survival of oligodendroglial cells consisted of neurobasal medium supplemented with FCS plus growth factors (EGF and FGF-2). Cells adhered to the substrate and started to form the cytoplasmic outgrowths. In three days, the first morphological differences between the differentiated cells became evident. The astroglial cells were flattened with few pale processes while oligodendroglial progenitors displayed few elongated thin processes. Neuronal cells were distinguishable later, by day 7. Because they looked like multiprocess bearing cells, they were initially similar to developing oligodendroglial cells in their morphology. Nevertheless, their amount was quite small. In a discontinuous monolayer, oligodendroglial cells were localized to empty spaces left between astroglial cells. Oligodendroglial cells with terminal outlying processes were in contact with peripheral edges of astroglial cells. The cells were still proliferating and in a week they formed a monolayer (Fig. 1C). The cells that
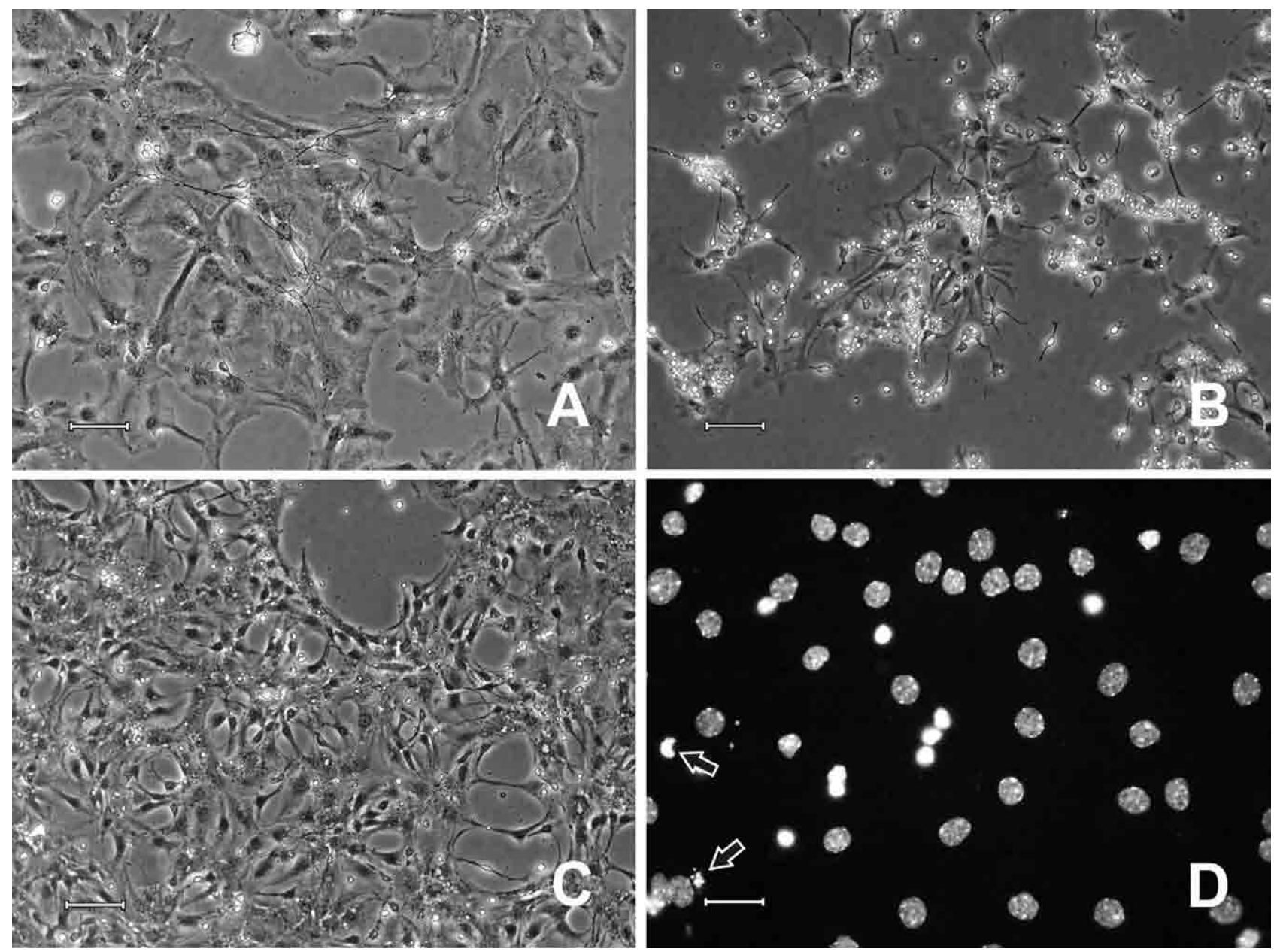

Fig. 1: Differentiation assays. A) Cells in serum-supplemented medium without growth factors differentiated mainly into astroglial monolayer. Few cells with bipolar or multipolar morphology survived on top of astrocytic cells. B) Exposure of dissociated neurospheral cells to serum-free medium supplemented with retinoic acid triggered massive cell death. C) Cells grown in cultivation medium supplemented with serum plus growth factors were rich in cells which differentiated preferentially into astroglia and oligodendroglia. Astroglia grown in media containing (C) growth factors have numerous slim processes, whereas astroglia in medium not containing factors (B) remain flattened and sometimes do not extend processes at all. D) The total amount of cells in a culture is assessed from the number of cell nuclei counterstained with DAPI; in medium supplemented with growth factors, cell nuclei that show features of cell death are scarcely visible (arrows). Scale bar: $20 \mu \mathrm{m}$. 

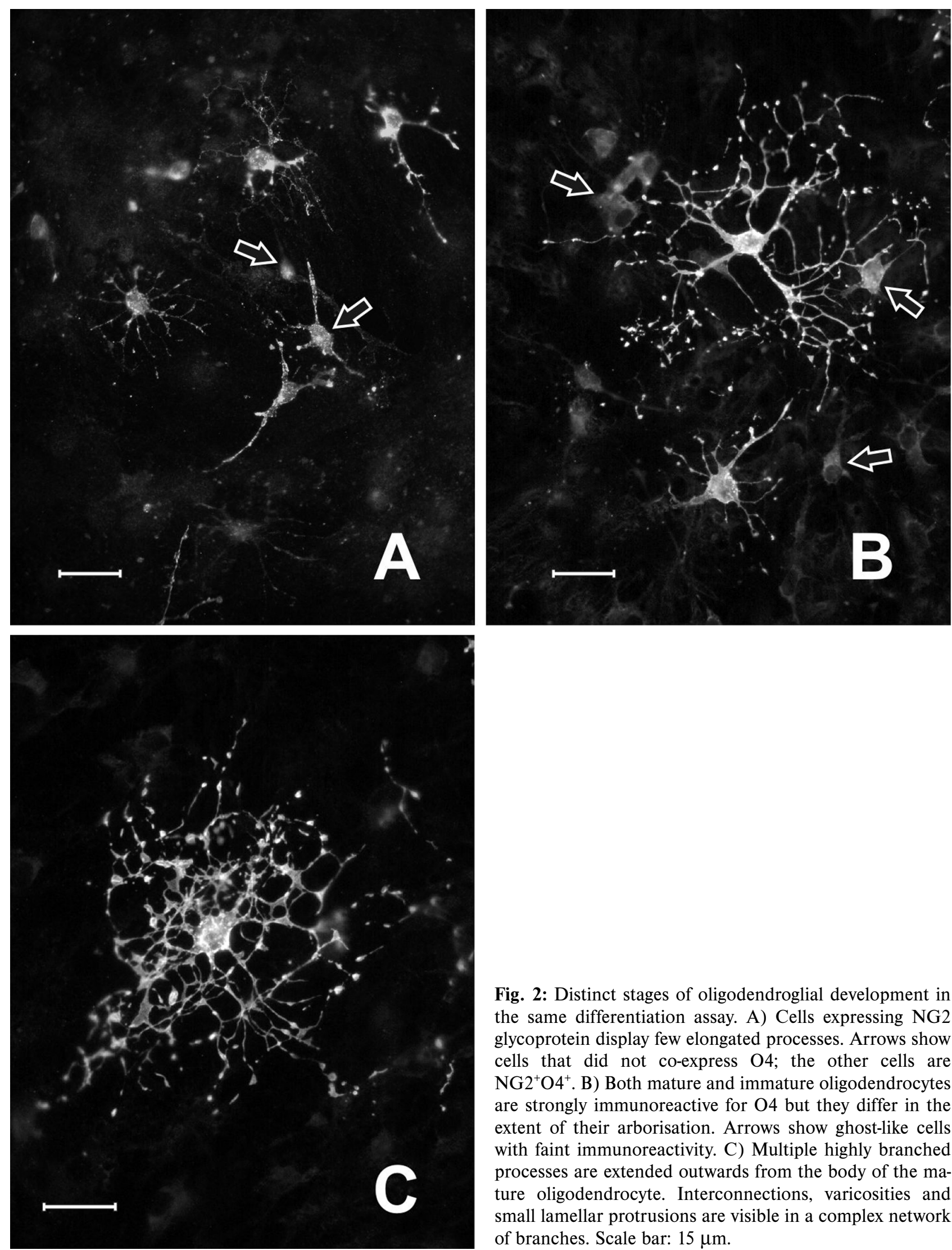

Fig. 2: Distinct stages of oligodendroglial development in the same differentiation assay. A) Cells expressing NG2 glycoprotein display few elongated processes. Arrows show cells that did not co-express $\mathrm{O} 4$; the other cells are $\mathrm{NG}_{2}^{+} \mathrm{O}^{+}$. B) Both mature and immature oligodendrocytes are strongly immunoreactive for $\mathrm{O} 4$ but they differ in the extent of their arborisation. Arrows show ghost-like cells with faint immunoreactivity. C) Multiple highly branched processes are extended outwards from the body of the mature oligodendrocyte. Interconnections, varicosities and small lamellar protrusions are visible in a complex network of branches. Scale bar: $15 \mu \mathrm{m}$. 
underwent cell death were rarely detectable (Fig. 1D). Over this period, the number of oligodendroglial cells also increased and they grew on a surface of astroglial monolayer. Under the phase contrast, neuronal cells were not distinguishable in a continuous monolayer. Oligodendroglial progenitors were detected according to their typical morphology. Their polygonal irregular nucleus protruded above the level of other cells and reflected light in a whitish halo that surrounded the body. These cells were clustered in small and separated groups.

Immunocytochemical examination performed after two weeks permitted to identify distinct subpopulations of cells that grew in the culture. However, almost a third of cells in the culture did not show immunoreactivity for any marker used in this study. Approximately two thirds of cells expressing cell-specific markers were represented by GFAP ${ }^{+}$ astrocytes and one third expressed oligodendroglial markers NG2 and/or O4. Only sporadic cells expressed $\beta$-III tubulin. Oligodendroglial cells exhibited characteristic multiprocess morphology and did not stain for markers of astroglial and neuronal cells. $\mathrm{NG}^{+} \mathrm{O}^{-}$- oligodendrocyte progenitors had only 1-5 primary processes that branched only occasionally (Fig. 2A). $\mathrm{NG}^{+} \mathrm{O}^{-}$preoligodendrocytes with small oval nucleus exhibited 4-10 primary processes that regularly sent secondary branches. A total amount of their secondary processes were twice as numerous as their primary processes. Occasionally, secondary processes formed tertiary branches. Another subset of oligodendroglial cells was labelled with $\mathrm{O} 4$ epitope but not with NG2. Although these immature and mature oligodendrocytes strongly expressed $\mathrm{O} 4$ in their perikaryon and processes, the adjacent areas in the culture also contained smaller cells that expressed $\mathrm{O} 4$ only faintly and looked like ghosts (arrows in Fig. 2B). They were more numerous than $\mathrm{O}^{+}$oligodendrocytes (the ratio of their occurrence was 1:2-3), displayed short processes that branched rarely and were clustered in small groups. On the contrary, oligodendrocytes that expressed high levels of $\mathrm{O} 4$ formed a complex network with their cytoplasmic processes. The oligodendrocytes displayed 7-16 stout processes that formed 28-77 secondary processes in all. These were further divided into 21-71 tertiary processes that further branched in 5-52 quaternary processes, which sometimes formed branches of higher orders. Some secondary and higher processes made interconnections that contributed to the complexity of the arborisation of mature oligodendrocytes. Moreover, some oligodendrocytes began to make cytoplasmic protrusions and varicosities (Fig. 2C). These cells covered with their processes small areas of several underlying cells and formed non-overlapping field domains.

\section{Discussion}

The neurosphere assay that is used for the propagation of neural SCs in vitro was developed for maintenance of SCs in an undifferentiated state (24). To prevent cell diffe- rentiation in the assay the cells are not allowed to adhere to cultivation surfaces and they are stimulated with mitogenic growth factors EGF and/or FGF-2 to proliferate (9, 24, 27, 29). The third condition required for a long term cultivation of neural SCs involves regular neurosphere dissociation. Without dissociation, the differentiated progeny appears in time within the neurospheres. In our previous studies, we documented that cells inside non-passaged neurospheres were able to undergo terminal differentiation and produce neurons that made interneuronal axodendritic synapses, astrocytes with processes rich in intermediate filaments and oligodendrocytes that formed myelin sheaths around adjacent axons $(16,18,19)$.

To document multipotency of neural SCs and their ability to produce differentiated phenotypes, the mitogenic growth factors are removed from the neurosphere cultivation medium and cells are allowed to adhere to suitable adhesive substrates. The cultivation conditions may involve lots of modifications; for example, the different nature of adhesive substrates, cell density, the presence or absence of fetal calf serum, the addition of differentiation factors, hormones or growth factors etc. All these factors affect cell differentiation and influence the ratio of resulting cell types $(5,11,14,17,32)$. In the present study, we compared the efficacy in the production of differentiated phenotypes under three distinct cultivation conditions using the same adhesive substrate. First, the cell differentiation was triggered by cultivation in a medium where growth factors were substituted with fetal calf serum. A change in cultivation condition resulted in cell death that reduced the numbers of cells that differentiated. Relatively resistant astroglial cells that function in the CNS as major supporting elements were able to survive for more than two weeks and formed a supporting monolayer to which other sporadic differentiated cell types were anchored. In the second group of experiments, differentiation was induced by the addition of retinoic acid, a potent differentiation factor, to a serum-free medium. Its mechanism of action is well known and involves the stimulation of specific nuclear retinoic acid receptors and retinoid X receptors (e.g. 23). Retinoic acid is used to trigger neurogenic differentiation in embryonic SCs (8). As a result, the treatment induced rapid differentiation that was, however, followed by robust cell death, saving cells that grew in the astrocytic monolayer.

In the last experiment, differentiation was induced by cultivation of cells in a medium containing both serum and growth factors EGF plus FGF-2. These factors, supporting a survival and proliferation of neural SCs, prevented the massive cell death that occurred in other conditions and stimulated proliferation of neural SC progeny (21). Neural SCs are not the only neural cells that express receptor for EGF or FGF-2. Moreover, the affinity of membrane receptors to growth factors on these cells may change throughout the development. The early embryonic brain tissue expresses low numbers of high-affinity EGF receptors, which are capable of responding to very low concentrations of EGF. 
During late gestation, the number of the receptors increased as gestation advanced but their apparent affinities for EGF declined, which could be correlated with differing roles of the receptors as development proceeds from tissue growth to tissue differentiation (1). In fetal neural cells, EGF stimulates differentiation of astrocytes and oligodendrocytes as demonstrated by an increase in glutamine synthetase and 2',3'-cyclic nucleotide 3'-phosphohydrolase (10). Furthermore, EGF can regulate the development of at least some mature neural cells, since in the mammalian CNS, receptors for EGF were identified in the plasma membrane of mature astrocytes (about $10^{4}-10^{5}$ receptor molecules per cell) and oligodendrocytes (between $6 \times 10^{3}-10^{4}$ receptors/ cell), as reviewed by Lakshmanan et al. (15) and Morrison (20). Expression of receptors for FGF on cells of oligodendroglial cell lineage reflects the ability of FGF-2 to regulate growth of the relevant subpopulations (3). FGF-2 stimulates proliferation of oligodendrocyte progenitors and regulates their differentiation into the preoligodendrocyte $(4,7)$. Moreover, FGF-2 was reported to convert bipotent oligodendrocyte progenitors to multipotent stem cells (13). In harmony with the above described actions that EGF and FGF-2 exert on cells of astroglial and oligodendroglial cell lineages, the differentiation assay contained mainly astrocytes and oligodendrocytes.

The amount of cells belonging to oligodendroglial cell lineage was relatively high. These cells comprised a third of all differentiated cells, which are quantities larger than mentioned other investigators; a usual outcome of oligodendroglial cells after spontaneous differentiation represents less than $3 \%(14,27,29,30)$. Oligodendroglial cells observed in our cultures were found at different stages of their development. The cultures contained oligodendroglial progenitors, preoligodendrocytes, immature and mature oligodendrocytes. Because the normal development of oligodendroglia occurs according to a well-defined lineage with the four successive stages (reviewed e.g. in 22), the concurrent presence of all these stages in our culture indicated that these cells derived from neural SCs in vitro developed physiologically in a stepwise process. Cells in distinct phases of their development were recognized according to their characteristic morphology and antigenic profile. Oligodendrocyte progenitors were identified as cells expressing chondroitin sulphate proteoglycan NG2 and possessing few primary processes that branched only occasionally. Such morphology correlates well with the proliferative potential of these cells and their capacity for migration. These progenitors were immunonegative for $\mathrm{O} 4$ epitope (sulphatides/seminolipids), although a faint immunoreactivity of ghost-like cells (Fig. 2B) might comprise late stages of progenitor cells. A strong immunoreactivity for $\mathrm{O} 4$ is characteristic of preoligodendrocytes and oligodendrocytes. Preoligodendrocytes were identified by co-expression of NG2 proteoglycan. These cells were still mitotically active and therefore their arborisation was not large; the cells formed low numbers of primary processes that branched and occasionally gave rise to tiny tertiary processes. $\mathrm{O}^{+}$ oligodendrocytes did not stain for NG2 and displayed multiple stout processes, which divided into secondary, tertiary and quaternary branches. Some branches anastomosed with others. This way the oligodendrocytes acquired a highly complex dendritic morphology with elaborated process network resembling a spider web. In areas containing large accumulation of mature oligodendrocytes, the underlying cells in the monolayer were contacted by ramified fine processes of overlying $\mathrm{O}^{+}$cells. Although the surface of a monolayer was almost entirely covered with an extensive process network of $\mathrm{O}^{+}$cells, the process field domains of neighboring oligodendrocytes did not overlap. Such highly complex morphology correlates to functionally developing oligodendrocytes. In the gray matter of the CNS, these cells occupy distinct domains and contact lots of cells with their multiple processes. The utmost stage of differentiation of oligodendrocytes observed in our cultures was characteristic with the formation of multiple varicosities along their highly branched processes and occasional flattened protrusions. The presence of these structures indicates a readiness of oligodendrocytes for the formation of the myelin sheaths. Although the myelin sheaths do not appear in the culture, the capacity of oligodendroglial cells produced in vitro to form the lamellar structures was documented after their transplantation to dysmyelinated brains (2).

Results of experiments described in this study document to what extent cultivation conditions affect the numbers and phenotypes of cells that are produced in vitro after plating equal initial neural stem cells. The addition of EGF and FGF-2 to a serum-supplemented medium used to induce cell differentiation elevates the numbers of surviving cells and at the same time allows successive differentiation of oligodendroglial cells according to the normal developmental pattern. Moreover, oligodendrocytes that arise under these conditions bear all the features of physiological maturation. All these data give evidence that neural SCs represent an ideal source of multipotent cells for in vitro developmental studies and the production of oligodendroglial cells

\section{Acknowledgement}

The authors are thankful to Mrs. Hana Hollerová for her skillful technical assistance.

This work was supported by the research project No. NR/7969-3 from Internal Grant Agency of Ministry of Health, Czech Republic.

\section{Reference}

1. Adamson ED, Meek J. The ontogeny of epidermal growth factor receptors during mouse development. Dev Biol 1984;103:62-70.

2. Ader M, Schachner M, Bartsch U. Integration and differentiation of neural stem cells after transplantation into the dysmyelinated central nervous system of adult mice. Eur J Neurosci 2004;20:1205-10.

3. Bansal R, Pfeiffer SE. Regulation of FGF receptors in the oligodendrocyte lineage. Mol Cell Neurosci 1996;7:263-75.

4. Bansal R, Pfeiffer SE. Regulation of oligodendrocyte differentiation by fibroblast growth factor. Adv Exp Med Biol 1997;429:69-77. 
5. Chiang YH, Silani V, Zhou FC. Morphological differentiation of astroglial progenitor cells from EGF-responsive neurospheres in response to fetal calf serum, basic fibroblast growth factor, and retinol. Cell Transplantation 1996;5:179-89.

6. Engstrom CM, Demers D, Dooner M et al. A method for clonal analysis of epidermal growth factor-responsive neural progenitors. J Neurosci Meth 2002;117 $111-21$

7. Gard AL, Pfeiffer SE. Two proliferative stages of the oligodendrocyte lineage $\left(\mathrm{A} 2 \mathrm{~B}^{+} \mathrm{O}^{-}\right.$and $\mathrm{O}^{+} \mathrm{GalC}^{-}$) under different mitogenic control. Neuron 1990 5:615-25.

8. Glaser T, Brustle O. Retinoic acid induction of ES-cell-derived neurons: the radial glia connection. Trends Neurosci 2005;28:397-400.

9. Gritti A, Cova L, Parati EA, Galli R, Vescovi AL. Basic fibroblast growth factor supports the proliferation of epidermal growth factor-generated neuronal precursor cells of the adult mouse CNS. Neurosci Lett 1995;185:151-4.

10. Honegger P, Guentert-Lauber B. Epidermal growth factor (EGF) stimulation of cultured brain cells I. Enhancement of the developmental increase in glial enzymatic activity. Dev Brain Res 1983;11:245-51.

11. Johe KK, Hazel TG, Muller T, Dugich-Djordjevic MM, McKay RDG. Single factors direct the differentiation. Genes Dev 1996;10:3129-40.

12. Karbanová J, Mokrý J, Kotingová L. Neural stem cells transplanted into intact brains as neurospheres form solid grafts composed of neurons, astrocytes and oligodendrocyte precursors. Biomed Papers 2004;148:217-20.

13. Kondo T, Raff M. Oligodendrocyte precursor cells reprogrammed to become multipotential CNS stem cells. Science 2000;289:1754-7.

14. Lachyankar MB, Condon PJ, Quesenberry PJ, Litofsky NS, Recht LD, Ross AH Embryonic precursor cells that express trk receptors: induction of different cell fates by NGF, BDNF, NT-3, and CNTF. Exp Neurol 1997;144:350-60.

15. Lakshmanan J, Weichsel ME, Fisher DA. Epidermal growth factor in synaptosomal fractions of mouse cerebral cortex. J Neurochem 1986;46:1081-5.

16. Mokrý J. Neural precursor cells and their cultivation. Prague: Galén, 1999:171 $\mathrm{pp}$

17. Mokrý J, Karbanová J. Foetal mouse neural stem cells give rise to ependyma cells in vitro, Folia Biol (Prague) 2006;52:149-55.

18. Mokrý J, Šubrtová D, Němeček S. Differentiation of epidermal growth factor-responsive neural precursor cells within neurospheres. Acta Med (Hradec Králové) $1996 ; 39: 7-20$
19. Mokrý J, Šubrtová D, Němeček S. Microstructure of nonpassaged spheroids formed by EGF-responsive neural precursor cells in vitro. Elec J Pathol Histo 1999;5:992-1006.

20. Morrison R. Epidermal growth factor: Structure, expression, and functions in the central nervous system. In: Loughlin SE, Fallon JH, eds. Neurotrophic factors. London: Academic Press Inc, 1993:339-357.

21. Mytilineou C, Park TH, Shen J. Epidermal growth factor-induced survival and proliferation of neuronal precursor cells from embryonic rat mesencephalon. Neurosci Lett 1992;135:62-6.

22. Pazour J, Mokrý J. Stem cell therapy for demyelinating disorders. Acta Med (Hradec Králové) 2006;49: 79-85

23. Peck GL, Di Giovanna JJ. Synthetic retinoids in dermatology. In: M. B. Sporn MB, Roberts AB, Goodman. DS, eds. The retinoids: biology, chemistry and medicine. New York: Raven Press, 1994:631-658.

24. Reynolds BA, Tetzlaff W, Weiss S. A multipotent EGF-responsive striatal embryonic progenitor cell produces neurons and astrocytes. J Neurosci 1992;12:4565-74.

25. Reynolds BA, Weiss S. Clonal and population analysis demonstrate that an EGFresponsive mammalian embryonic CNS precursor is a stem cell. Dev Biol 1996;175:1-13.

26. Spradling A, Drummond-Barbosa D, Kai T. Stem cells find their niche. Nature 2001;414:98-104.

27. Tropepe M., Sibilia M, Ciruna BG, Rossant J, Wagner EF, Kooy D. Distinct neural stem cells proliferate in response to EGF and FGF in the developing mouse telencephalon. Dev Biol 1999;208:166-88.

28. Uchida N, Buck DW, He D et al. Direct isolation of human central nervous system stem cells. Proc Natl Acad Sci USA 2000;97:14720-5.

29. Vescovi AL, Parati EA, Gritti A. Isolation and cloning of multipotential stem cells from the embryonic human CNS and establishment of transplantable human neural stem cell lines by epigenetic stimulation. Exp Neurol 1999;156:71-83.

30. Wachs FP, Couillard-Despres S, Engelhardt $M$ et al. High efficacy of clonal growth and expansion of adult neural stem cells. Lab Invest 2003;83:949-62.

31. Weissman IL. Stem cells: units of development, units of regeneration, and units in evolution. Cell 2000;100:157-67.

32. Whittemore SR, Morassutti DJ, Walters WM, Liu RH, Magnuson DSK. Mitogen and substrate differentially affect the lineage restriction of adult rat subventricular zone neural precursor cell populations. Exp Cell Res 1999;252:75-95.

Submitted January 2007.

Accepted January 2007.

\section{Corresponding author:}

Prof. MUDr. Jaroslav Mokrý, Ph.D., Charles University in Prague, Faculty of Medicine in Hradec Králové, Department of Histology and Embryology, Šimkova 870, 50038 Hradec Králové, Czech Republic, e-mail: mokry@lfhk.cuni.cz 